\title{
Interband transitions and critical points of single crystal thoria compared to urania
}

\author{
Christina Dugan ${ }^{1, *}$, Lu Wang ${ }^{2}$, Kai Zhang ${ }^{2}$, J. Matthew Mann ${ }^{3}$, Martin Kimani ${ }^{4}$, Wai-Ning Mei ${ }^{5}$ Peter Dowben \\ $6, *$, and James Petrosky ${ }^{1}$ \\ 1 Department of Engineering Physics, Air Force Institute of Technology, 2950 Hobson Way, Wright-Patterson Air Force \\ Base, Ohio 45433-7765, U.S.A. \\ 2 CAS Key Lab of Materials for Energy Conversion, Department of Materials Science and Engineering, Uni- \\ versity of Science and Technology of China, Hefei, Anhui 230026, China. \\ 3 Air Force Research Laboratory, Wright-Patterson Air Force Base, OH 45433-7765, U.S.A. \\ 4 KBRwyle, Dayton, Ohio 45431, U.S.A. \\ 5 Department of Physics, University of Nebraska at Omaha, 60th and Dodge Streets, Omaha NE 68182-0266, \\ U.S.A. \\ 6 Department of Physics and Astronomy, Theodore Jorgensen Hall, 855 North 16th Street, University of \\ Nebraska-Lincoln, Lincoln, NE 68588-0299, U.S.A. \\ * Correspondence: Christina.Dugan@afit.edu, Tel.: 937-255-3636 x4407; pdowben@unl.edu; Tel.: 402-472-9838
}

Featured Application: Since its discovery in the late 1700's, urania $\left(\mathrm{UO}_{2}\right)$ has been used in a variety of applications including early application as a bright pigment amendment for ceramics and as a hue absorbent in lenses. Later, when nuclear properties were discovered, urania gained interest as a fissile fuel and radioactive source in the power industry. While urania and thoria are generally considered wide band gap semiconductors, there is potential application for urania as as a gamma insensitive integrated sole state neutron detector for national security and nuclear fuel management. The potential value of a urania-based semiconductor detector is based upon the large energy released from a fission event of $\sim \mathbf{1 8 0} \mathrm{MeVs}$. If the energy released by a neutron occurs in a diode junction, the current produced would be unique to the neutron capture event, making discrimination between gamma and neutron capture possible. Neutron spectral sensitivity, due to the differences in uranium isotopic cross section differences could be used to assess the neutron spectrum in a pixelated detector system. Additionally, such urania solid state detector also benefits from efficiencies not found in other neutron detectors, since the conversion material (uranium) is the same as the semiconducting material. Further, a neutron detection system could be envisioned in remote locations where the thermoelectric properties (or neutron voltaic properties) of urania could be used to make a self-powered system. The use of a urania based electronics can provide new capability as an integrated inventory system on unused nuclear fuel, without making significant changes to the nuclear performance. The potential to manufacture a circuit on a fuel pellet with RFID response circuitry would allow for remote inventory and identification of fuel pellets in transit or storage.

\begin{abstract}
The interband transitions of urania $\left(\mathrm{UO}_{2}\right)$ are validated independently through cathode luminescence of $\mathrm{UO}_{2}$. A picture emerges consistent with density functional theory. While theory is generally consistent with experiment, it is evident that the choice of functional can significantly alter the band gap and some details of the band structure, in particular at the conduction band minimum. Strictly $a b$ initio predictions of the optical properties of the actinide compounds, based on density functional theory alone continues to be somewhat elusive.
\end{abstract}

Keywords: cathode luminescence; urania; thoria; interband transitions

\section{Introduction}

The electronic properties of actinide dioxides, from $\mathrm{UO}_{2}$ to $\mathrm{AmO}_{2}$, includes the localized $5 f$ electronic states, whose strong on-site Coulomb repulsion interaction is often severely underestimated in density functional theory (DFT) calculations [1-6]. An accurate 
quantitative description of the optical spectra requires theory beyond the independent particle picture, yet qualitative agreement between theory and experiment can often be obtained on the level of DFT [7]. While the optical adsorption and reflectance spectra of semiconductors have been studied for several decades, similar work on actinide dioxides has been hampered by the absence of high quality single crystals [8,9].

Experimentally, Schoenes studied the incident light reflectivity off $\mathrm{UO}_{2}$ single crystals in the photon energy range of $0.03-13 \mathrm{eV}$, from which the complex dielectric function $e(w)=e_{1}(w)+i_{2}(w)$ has been derived [10]. This effort to extract the complex dielectric function has been repeated more recently by Siekhaus and J. Crowhurst [11] as well as Mock, Dugan and coworkers [12,13]. These have transitional charactertistics that are shifted significantly compared to reflectivity measurements by Schoenes [10]. Absorption of $\mathrm{UO}_{2}$ thin films, by optical transmission has also been measured [14], while the optical properties for both $\mathrm{ThO}_{2}$ and $\mathrm{UO}_{2}$ have been previously calculated [15] using the HeydScuseria-Ernzerhof (HSE) functional. The challenges of growing uranium oxide singlecrystals have been addressed with the fabrication of high-quality, single crystal actinide oxide samples using a hydrothermal synthesis growth technique [12,13,16-22]. This growth process has produced bulk single crystals of near-stoichiometric $\mathrm{UO}_{2}[12,13,17,20$ 22] and $\mathrm{ThO}_{2}[13,18,19,22]$. These improved single crystals make higher quality assessments of the $\mathrm{UO}_{2}$ and $\mathrm{ThO}_{2}$ optical and semiconductor properties possible, as defect contributions to the complex dielectric function are much suppressed [13]. In this study, we validate the observed interband transitions identified in variable angle ellipsometry $[12,13]$ with cathode luminescence and theory.

\section{Materials and Methods}

Single crystals of $\mathrm{UO}_{2}$ were grown by hydrothermal synthesis [12,13,16-22], as described in $[12,13,22]$. Further hydrothermal growth information is detailed in [16]. The $\mathrm{UO}_{2}$ crystals grown under these conditions have measured lattice parameters of $5.4703 \pm$ $0.0006 \AA$ indicating a stoichiometry near $\mathrm{UO}_{2.003}[23,24]$.

The cathodoluminescence system consists of a Kimball Physics EMG-12 electron gun powered by an EGPS-12 power supply, a vacuum system, a sample chamber with suitable optical ports, an optical system, a spectrometer, and a photomultiplier detector. The liquid nitrogen-cooled $\mathrm{UO}_{2}$ was placed at the focal point of the electron gun and the resulting cathodoluminescence signal from the sample was collected through a quartz window of the vacuum chamber, focused onto the entrance slit of the monochromator and then to the photomultiplier or a solid state detector. The electron beam was incident at an angle of approximately $45^{\circ}$ withan incident energy of $10 \mathrm{KeV}$ energy.

All the electronic band structure calculations were performed within the framework of spin-polarized plane-wave density functional theory (PW-DFT), implemented in the Vienna ab initio simulation package (VASP) [25,26]. The Perdew-Burke-Ernzerhof (PBE) functional and projector augmented wave (PAW) potentials were used [27-29]. An energy cutoff of $600 \mathrm{eV}$ was adopted for the plane-wave expansion of the electronic wave function. The Brillouin zone integration was sampled with $9 \times 9 \times 9 \mathrm{k}$-point mesh for bulk and $9 \times 9 \times 1$ k-point mesh for slab models. Geometry structures are relaxed until the force on each atom is less than $0.01 \mathrm{eV} / \AA$ and the energy convergence criteria of $10^{-7} \mathrm{eV}$ are met. Once the optimized structures were achieved, the hybrid functional, in the Heyd-ScuseriaErnzerhof (HSE06) [30] form, was used to give more accurate band gaps. The imaginary part of the frequency-dependent dielectric matrix was calculated based on the HSE06 ground states of $\mathrm{ThO}_{2}$ by using random phase approximation (RPA) [31,32].

When the DFT $+U$ method was used, a correlation energy correction Hubbard parameter $(\mathrm{U})$ was used with the value $U=2 \mathrm{eV}$ for $f$-states of Th atoms to provide the necessary correction for the on-site Coulomb interactions. Other work on thoria have used value in the region of $2 \mathrm{eV}$ to $4.0 \mathrm{eV}$ and while the precise value is difficult to ascertain without comparison with experiment, these $\mathrm{DFT}+\mathrm{U}$ calculations provided reasonable qualitative results as the calculated band gap of $\mathrm{ThO}_{2}$ bulk, in the $\mathrm{PBE}+\mathrm{U}$ functional, is consistent with most other theory efforts [15,33-37], as discussed below. 


\section{Interband transitions of $\mathrm{ThO}_{2}$ and $\mathrm{UO}_{2}$}

When the DFT $+U$ method was used, with a choice of $U=2 \mathrm{eV}$ for $f$-states of Th atoms, the calculated band gap is found to be $5.04 \mathrm{eV}$. Using the Heyd-Scuseria-Ernzerhof functional, to estimate the band gap more accurately, our calculations revealed that the ground states of bulk $\mathrm{ThO}_{2}$ are nonmagnetic, with a band gap of $6.12 \mathrm{eV}$, as seen in Figure 1. This is close to the $6.21 \mathrm{eV}$ value previously calculated [15]. It has also been shown that the Heyd-Scuseria-Ernzerhof band structure calculations, with spin-orbit coupling turned on, provides a band gap of $5.8 \mathrm{eV}$ [33], which is closer to the experimental value of $5.75 \mathrm{eV}$ [34] and our value of $5.4 \mathrm{eV}$ measured from variable angle ellipsometry. The HeydScuseria-Ernzerhof functional does, however, differ significantly from the calculated band structure obtained using Perdew, Burke, Ernzerhof (PBE), discussed below, and the band structures previously reported [34,35].

Figure 1. The band structure of bulk $\mathrm{ThO}_{2}$ using Heyd-Scuseria-Ernzerhof (HSE) hybrid functional. The calculated band gap is $6.12 \mathrm{eV}$ and the ground state is anti-ferromagnetic.

These band gaps are not the signature of surface effects. Density functional theory slab calculations indicates that $\mathrm{ThO}_{2}(001)$, with a Th atom termination, is not a spin polarized semiconductor, and in this approach, we are left with the suggestion that the band gap is small, of order $0.28 \mathrm{eV}$. $\mathrm{ThO}_{2}(100)$, with an $\mathrm{O}$ atom termination, is a ferromagnetic metal with $4.0 \mathrm{mв}$ magnetic moments for the supercell. Basically, by including the surface,

Band gap: $6.12 \mathrm{eV}$
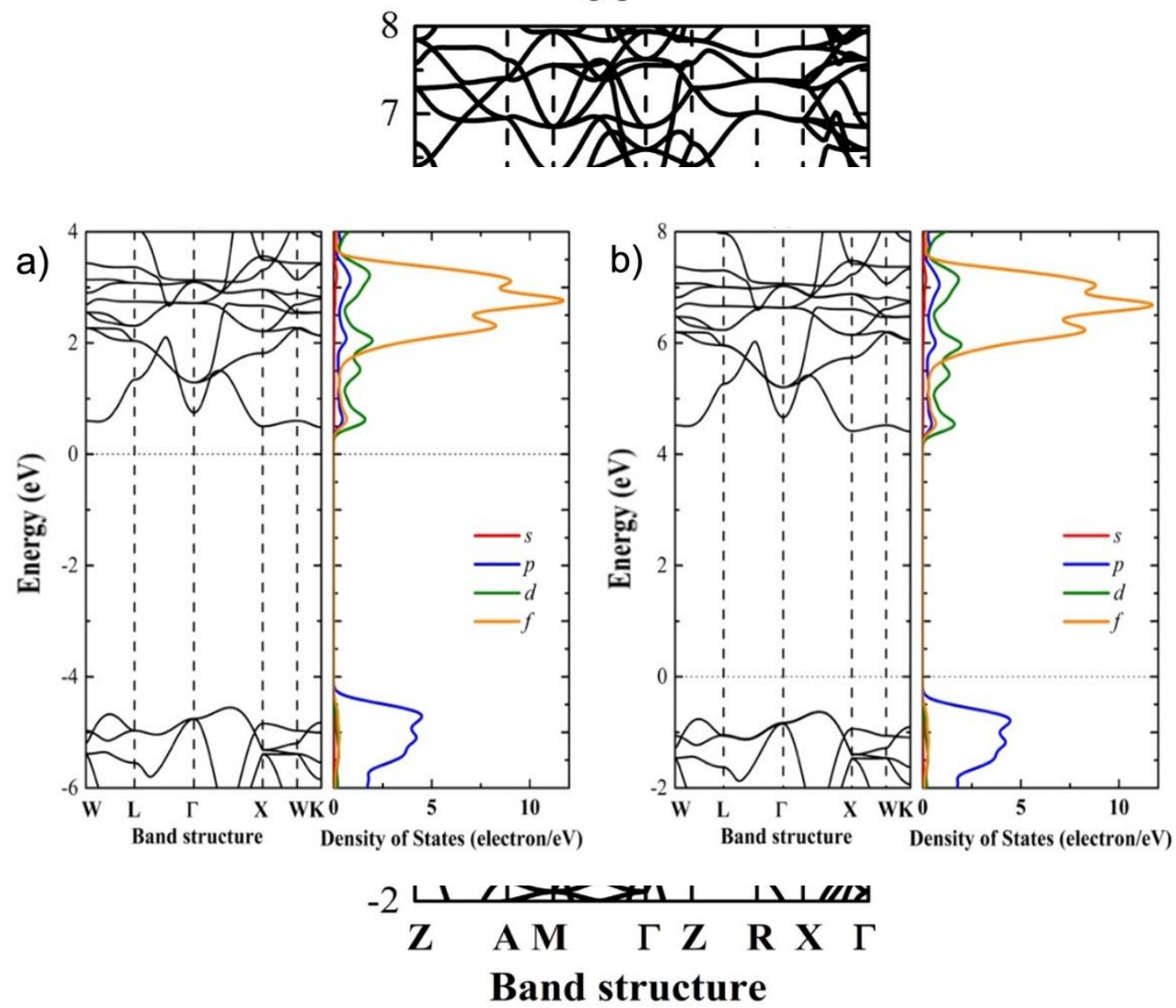

the $\mathrm{ThO}_{2}(001)$ band structure is characteristic of a semimetal, an n-type semimetal if the $\mathrm{ThO}_{2}(100)$ surface terminates with Th atoms, and a p-type semimetal if the $\mathrm{ThO}_{2}(001)$ surface terminates with Th atoms. The semimetal band gap has an indirect gap of about 2.7 $\mathrm{eV}$, and in any case, the predicted semimetal behavior of the surface is not what has been measured here. 
Figure 1. Structure and density of states of $\mathrm{ThO}_{2}$ by the (a) $\mathrm{PBE}+\mathrm{U}$ functional in the density mixing scheme and by (b) PBE+U function in the EDFT scheme. In both cases the estimatedband gap comes out to be $5.04 \mathrm{eV}$

The $\mathrm{ThO}_{2}$ band structure, calculated using the Perdew-Burke-Ernzerhof (PBE) functional with a correlation $\mathrm{U}=2 \mathrm{eV}$, as shown in Figure 2, is qualitatively similar to that calculated through the B3LYP functional [34] and the relativistic linear augmented-planewave (RLAPW) calculation of [35] for the valence band. This calculated band structure differs, however, significantly from the calculated band structure, using the B3LYP functional or RLAPW, on the conduction band side. One difference with the calculated band structure of [34,35], and the calculations shown here, is that the calculation of Figure 1 does have the conduction band minimum at the $\mathrm{L}$ point of the Brillouin zone, as is seen in [34]. For the pure $\mathrm{ThO}_{2}$ bulk, the band gap is $5.04 \mathrm{eV}$ in the $\mathrm{PBE}+\mathrm{U}$ functional, consistent with most other theory published to date [15,34-37]. A similar band gap was obtained with $\mathrm{PBE}+\mathrm{U}$ in the density mixing scheme and the EDFT scheme. This consistency is expected as the calculated band structures, within the same PBE functional plus a correlation energy $U$ from different convergence schemes, should converge to the same ground states. While the band structure is very similar, one approach makes $\mathrm{ThO}_{2}$ more p-type (Figure $2 \mathrm{~b}$ ) and the other makes $\mathrm{ThO}_{2}$ more n-type (Figure 2a). A key point that comes from comparing the band structures obtained from using different functionals is that the resulting calculated band structures differ and thus the choice of functional matters.

The calculated bulk dielectric function shown in Figure 3 for $\mathrm{ThO}_{2}$. Absorption and the optical response will occur for any symmetry and selection rule allowed transition at any place in the Brillouin zone, not just $\Gamma$, so long the transition itself has little or no momentum exchange, i.e. is $\Delta q=0$ transition. Basically, the low-lying critical points seen in the variable angle ellipsometry experiments of $\mathrm{ThO}_{2}$ [13] are qualitative reproduced in theory, though shifted to higher energies in the HSE theory, as summarized in Table 1. For PBE, the calculated bulk dielectric function provides agreement more in line with experiment, but also qualitatively similar to HSE. This means that the strongest contributions to the optical properties for $\mathrm{ThO}_{2}$ are the $\mathrm{p}-\mathrm{d}$ transitions in the region of $5-8 \mathrm{eV}$. This places the low lying critical points for $\mathrm{ThO}_{2}$ at $5.5 \mathrm{eV}$ (the $\Delta \mathrm{q}=0$ [p to d; e symmetry] transition in the $\varepsilon_{2}(\omega)$ part of the dielectric function for single crystalline $\mathrm{ThO}_{2}$, and the $\Delta \mathrm{q}=0$ [ $p$ to $d$; a1 symmetry] transition in the region of the center of the Brillouin zone) and at 6.8 $\mathrm{eV}$ (the combined $\mathrm{q}=0$ transitions [ $p$ to $d$; e symmetry] in the region of $\mathrm{W}$ and $\mathrm{K}$ of the Brillouin zone). These transitions are strongly Th $O 2 p \rightarrow T h 6 d / 5 f$ transitions for the most part, weight more Th $6 d$ in the final state for the transitions at lower energies and more and more Th $5 f$ in the final state for the transitions at energies in the region of 8 to $9 \mathrm{eV}$. 


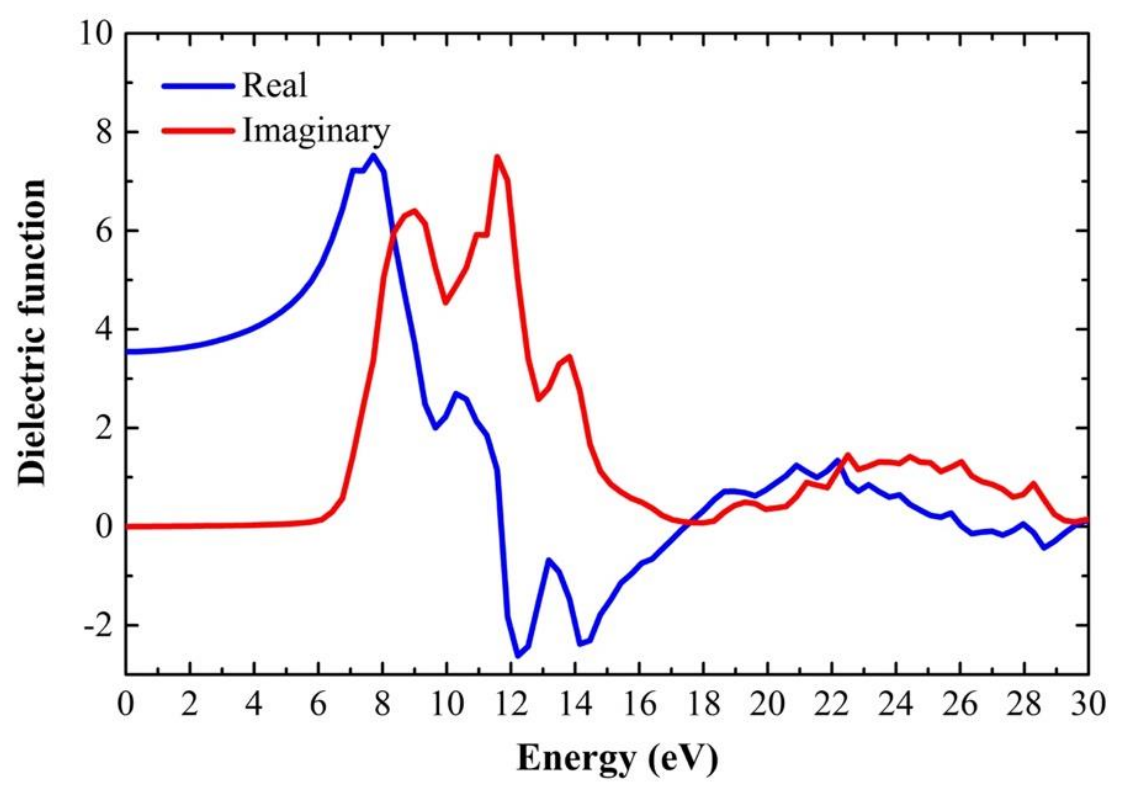

Figure 3. The calculated real (blue) and imaginary (red) parts of the $\mathrm{ThO}_{2}$ dielectric function.

Five critical points have been identified in the experimental spectra taken for both of the actinide oxides $\mathrm{UO}_{2}$ (table 2) and $\mathrm{ThO}_{2}$ (table 1) [13] and can be compared with both theory and cathodoluminescence, as discussed below. From theory, the two actinides present with a very similar oscillator pattern that appears to be compressed and shifted to higher energy for the $\mathrm{ThO}_{2}$, for the features below $10 \mathrm{eV}$. The imaginary part of the calculated dielectric response for the $\mathrm{ThO}_{2}$ has the first major feature at $9 \mathrm{eV}$, as seen in Figure 3. In the dielectric response calculated from the experimental variable angle spectroscopic ellipsometry [13], the first low energy peak appears at a lower energy of 8 $\mathrm{eV}$, consistent with the fact that the HSE functional estimates a larger band gap than is observed experimentally (Table 1 ).

In the case of $\mathrm{UO}_{2}$, the dielectric response derived from the experimental variable angle spectroscopic ellipsometry [12,13] has two peaks at low energy, at $2.0 \mathrm{eV}$ and 5.0 $\mathrm{eV}$ respectively. These low energy features are not as well resolved in theory, as seen in Figure 4, but as noted elsewhere [12], the band gaps generally agree with theory if the HSE functional is used, while the Perdew-Burke-Ernzerhof (PBE) functional grossly underestimates the band gap (as discussed in detail elsewhere [12]). What are peaks in the calculated imaginary part of the dielectric response for $\mathrm{UO}_{2}$ (Figure 4), appear only as higher energy shoulders in experiment [13] and as weak features in the cathodoluminescence. This suggests that experiment and theory do not agree as to the extent of the oscillator strength used to describe the critical points lower energies. 


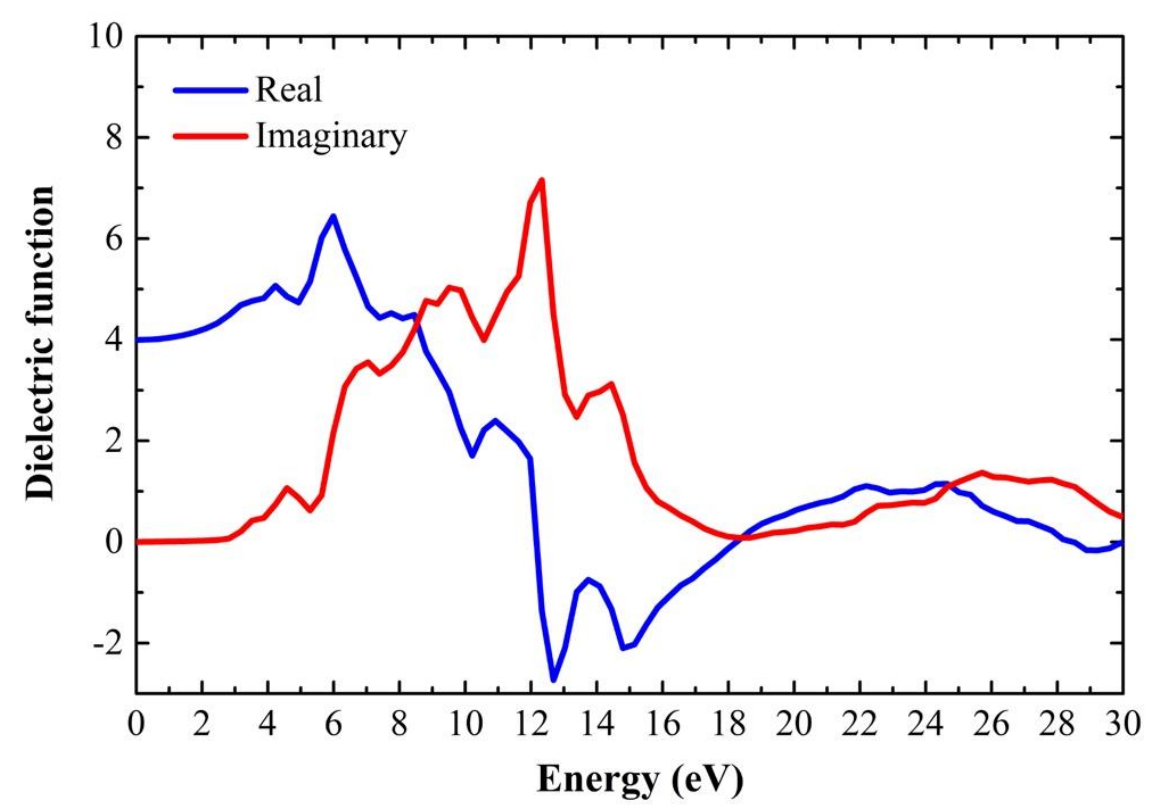

Figure 4. The calculated real (blue) and imaginary (red) parts of the $\mathrm{UO}_{2}$ dielectric function, using the HSE functional.

We observe that the critical points seen in $\mathrm{UO}_{2}$ are broader than their counterparts in $\mathrm{ThO}_{2}$ in the imaginary part of the dielectric response extracted from the experimental variable angle spectroscopic ellipsometry [13], but the reverse is true in theory. These trends are seen in the calculated real and imaginary parts of the dielectric function for $\mathrm{ThO}_{2}$ (Figure 3) and $\mathrm{UO}_{2}$ (Figure 4). For the features at roughly 10,12, 14 and $15 \mathrm{eV}$, thoria $\left(\mathrm{ThO}_{2}\right)$ and urania $\left(\mathrm{UO}_{2}\right)$ resemble each other as noted in [15]. The calculated optical properties of Figures 3 and 4 differ, but it should be noted that there is now a wealth of information on $\mathrm{UO}_{2}$, as summarized in Table 2, and the transition energies in variable angle ellipsometry previously measured [12,13], agree with the cathodoluminescence and theory. This means that the transitions can be assigned, based on the theory.

Table 1. Critical point energies for $\mathrm{ThO}_{2}$, obtained from variable angle ellipsometry (VASE) [13] compared to theory

\begin{tabular}{ccc}
\hline Experiment $(\mathrm{eV})^{1}$ & Theory $(\mathrm{eV})^{\mathbf{2}}$ & Assignment \\
\hline 5.4 & 6.1 & Th $\mathbf{6} \boldsymbol{d} \rightarrow O 2 p$ \\
6.3 & 7.2 & Th $\mathbf{6} \boldsymbol{d} \rightarrow O 2 p$ \\
7.5 & 7.7 & Th $\mathbf{6} \boldsymbol{d} / \mathbf{5} \boldsymbol{f} \rightarrow O 2 p$ \\
8.0 & 9.0 & Th $\mathbf{5} \boldsymbol{f} \rightarrow O 2 p$ \\
8.8 & - & \\
\hline
\end{tabular}

1. Experimentally determined energy values from VASE, taken from [13]

2. HSE theoretically determined energy values. 
Table 2. Critical point energies for $\mathrm{UO}_{2}$, obtained from variable angle ellipsometry (VASE) [13] and cathode luminescence compared to theory

\begin{tabular}{|c|c|c|c|}
\hline $\begin{array}{c}\text { Experiment }(\mathrm{eV})^{1} \\
\text { VASE }\end{array}$ & $\begin{array}{c}\text { Experiment }(\mathrm{eV})^{2} \\
\mathrm{CL}\end{array}$ & Theory $(\mathrm{eV})^{3}$ & Assignment \\
\hline \multirow[t]{2}{*}{2.0} & 2.4 & 2.2 & $U 5 f \rightarrow U 5 f$ \\
\hline & 2.6 & & \\
\hline \multirow[t]{2}{*}{2.6} & & 3.5 & $U 5 f \rightarrow U 5 f$ \\
\hline & 2.9 & & \\
\hline 5.0 & 3.8 & 4.6 & $U 5 f \rightarrow U 6 d$ \\
\hline 6.3 & 6.1 & 6.0 & $O 2 p \rightarrow \boldsymbol{U} \mathbf{6} d / \mathbf{5} f$ \\
\hline 6.9 & 6.8 & 6.9 & $O 2 p \rightarrow \boldsymbol{U} \mathbf{6} d / \mathbf{5} f$ \\
\hline
\end{tabular}

\subsection{Cathodoluminescence}

Figure 5 displays the cathodoluminescence, taken at $10 \mathrm{keV}$, for a single crystalline $\mathrm{UO}_{2}$ sample grown by hydrothermal synthesis. The values given in Table 2 generally agree with the optical transition values extracted from the variable angle spectroscopic ellipsometry [12,13]. An experimentally determined band gap of $2.37 \mathrm{eV}$, from cathodoluminescence, is also consistent with theoretically predicted band gap of $2.19 \mathrm{eV}$ [12]. This value of $2.37 \mathrm{eV}$ is larger than previously found by VASE (Table 1) and discussed at length elsewhere [12,13]. As there is no initial state photohole, the luminescence corresponding to the lowest unoccupied state to highest occupied state transition is expected to be larger, as is the case with the value determined from VASE. The very low luminescence for the transitions corresponding to the band gap is the result of being a selection rule forbidden transition.

The fact that the cathodoluminescence features are generally very weak, especially for the luminescence corresponding to the lowest unoccupied state to highest occupied state transition for single crystal $\mathrm{UO}_{2}$, is because this transition is a selection rule forbidden transition. A $U 5 f \rightarrow U 5 f$ transition does not obey the required $\Delta l= \pm 1$ for an optical transition. The $\mathrm{U} 6 d / 5 f \rightarrow \mathrm{O} 2 p$ luminescence transitions are also expected to be weak as they are extra-atomic transitions, as is observed here. As seen in Figure 5, the strongest luminescence feature is the on-site, selection rule allowed $\mathrm{U} 6 d \rightarrow \mathrm{U} 5 f$ transition (the reverse of the transition in variable angle ellipsometry previously measured [13]). This very strong cathodoluminescence feature at $3.8 \mathrm{eV}$ appears at a lower energy than predicted by theory or seen in the variable angle ellipsometry previously measured [13] or predicted by theory, indicative of extensive hybridization in the band structure. These transition assignments are, nonetheless, generally consistent not only with theory [10], but also combined photoemission and inverse photoemission studies of $\mathrm{UO}_{2}$ [38]. This, in turn, implies that $\mathrm{UO}_{2}$ and $\mathrm{ThO}_{2}$ are indeed strongly correlated systems with correlation energies in the region of $6 \mathrm{eV}$ for $\mathrm{ThO}_{2}$ [34] and 4-5 eV for $\mathrm{UO}_{2}[39,40]$. 


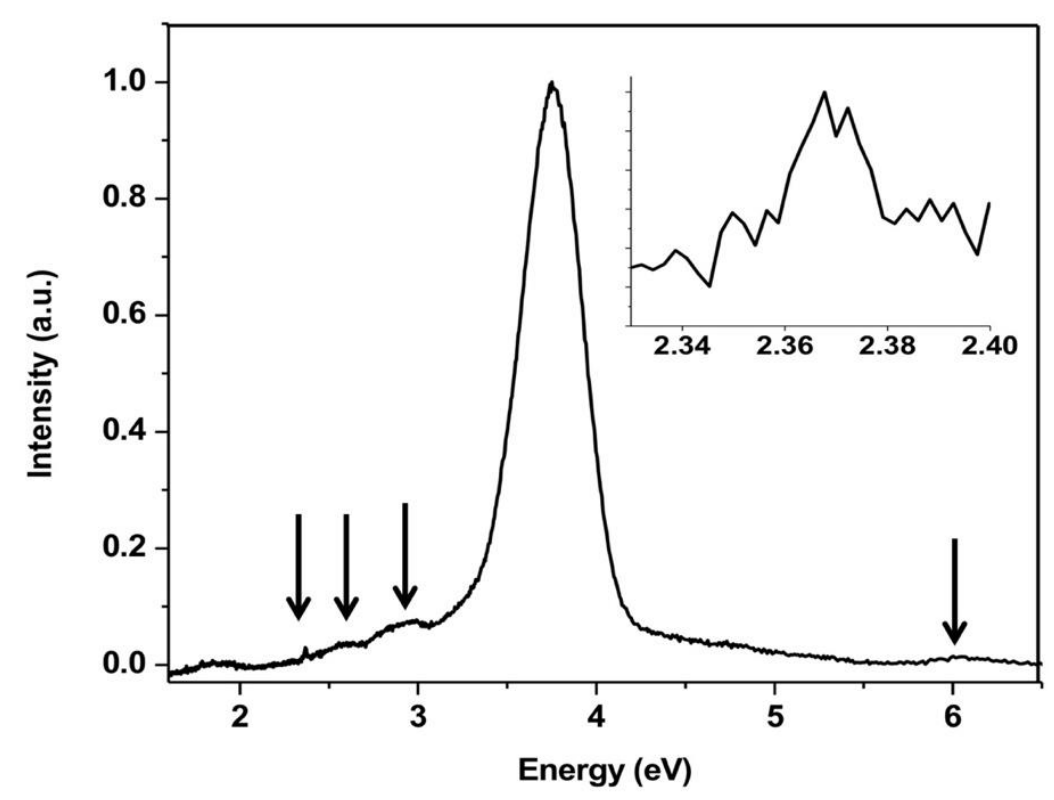

Figure 5. The optical transitions for single crystal $\mathrm{UO}_{2}$, measured from cathodoluminescence. The incident electron energy is $10 \mathrm{keV}$ to avoid surface effects. The weaker transitions, are indicated by arrows, and that corresponding to the lowest unoccupied state to highest occupied state transition is shown in the inset.

\section{Conclusions}

There is a consistent picture of the electronic structure, from experiment, that is not shared among the many theoretical band structure calculations. As noted elsewhere [38], when it comes theory, the final arbiter of successful theoretical band structure calculations presently appears to be in agreement with the experiment results for the actinide oxides. As sample quality improves, the difference between experiments and theory is increasingly diminished, although the role of screening does appear to have a profound effect on the experiment. Surface effects, not discussed here, are expected to be a persistent problem and caution is needed in interpreting experimental results with a strong surface contribution. For example, the photoelectric work function of the (111) hydrothermally grown $\mathrm{UO}_{2}$ was measured at $3-4 \mathrm{eV}$ [20], and $3.19 \pm 0.03 \mathrm{eV}$ [41]. More recently, the photoelectric determined work function of nearly stoichiometric hydrothermally grown $\mathrm{UO}_{2}(111)$ and (100) was measured to be $6.28 \pm 0.36 \mathrm{eV}$ and $5.80 \pm 0.36 \mathrm{eV}$, respectively [21]. This range of experimental values reduces confidence in key parameters associated with these materials. But, as shown in this paper, that confidence builds as theory and experiment begin to merge.

Supplementary Materials: Not Applicable.

Author Contributions: Conceptualization, P. D., C. D., and W. M.; methodology, P. D., C. D., J. M., W. M. and J. P.; software, L. W., K. Z., W. M. and P. D.; validation, P. D. and C. D..; formal analysis, P. D., L. W. and W. M.; investigation, M. K., L. W., K. Z., C. D., and P. D.; resources, J. P. and M. K.; writing-original draft preparation, P. D.; writing-review and editing, P. D., J. P. and C. D.; project administration, P. D. and J. P.; funding acquisition, P. D., J. M. and J. P. All authors have read and agreed to the published version of the manuscript.

Funding: This work was supported in part by the National Science Foundation through the EPSCoR RII Track-1: Emergent Quantum Materials and Technologies (EQUATE), Award OIA-2044049. This work was also supported in part by the Air Force Office of Scientific Research under award FA955018-1-0360, and by the Defense Threat Reduction Agency (Grant No. HDTRA1-14-1-0041), and the Domestic Nuclear Detection Office of the Department of Homeland Security (Grant No. HSHQDC14X00089). J. M. M. was supported by the Center for Thermal Energy Transport under 
Irradiation, an Energy Frontier Research Center funded by the U.S. Department of Energy, Office of Science, Office of Basic Energy Sciences.

Institutional Review Board Statement: Not applicable.

Informed Consent Statement: Not applicable.

Data Availability Statement: There are no relevant statements for this research.

Acknowledgments: Thanks to all the participants who promoted the successful completion of this article.

Conflicts of Interest: The authors declare no conflict of interest. The views expressed in this article are those of the authors and do not reflect the official policy or position of the United States Air Force, Department of Defense, or the U.S. Government.

\section{References}

1. Boettger, J.C.; Ray, A.K. Fully relativistic density functional calculations on hydroxylated actinide oxide surfaces. Int. J. Quant. Chem. 2000, 80, 824.

2. Boettger, J.C.; Ray, A.K. All-electron LCGTO calculations for uranium dioxide. Int. J. Quant. Chem. 2002, 90, 1470.

3. Jomard, G.; Amadon, B.; Bottin, F. Torrent. M. Structural, thermodynamic, and electronic properties of plutonium oxides from first principles. Phys. Rev. B. 2002, 78, 075125.

4. Zhang, P.; Wang, B.T.; Zhao, X.G. Ground-state properties and high-pressure behavior of plutonium dioxide: Density functional theory calculations. Phys. Rev. B. 2010, 82, 144110.

5. Wang, B.T.; Shi, H.; Li, W; Zhang, P. First-principles LDA+U and GGA+U study of neptunium dioxide. Phys. Rev. B. 2010, 81, 045119.

6. Lu, Y.; Yang, Y.; Zheng, F.W.; B.T. Wang, B.T.; Zhang, P. Electronic, mechanical, and thermodynamic properties of americium dioxide. J. Nucl. Mater. 2013, 441, 411.

7. Gajdoš, M.; Hummer, K.; Kresse, G.; Furthmuiller, J.; Bechstedt F. Linear optical properties in the projector-augmented wave methodology. Phys. Rev. B. 2006, 73, 045112.

8. Meek, T. T.; v. Roedern, B. Semiconductor devices fabricated from actinide oxides. Vacuum. 2008, 83, 226-228.

9. Kruschwitz, C.; S. Mukhopadhyay, S.; Schwellenbach, D.; Meek, T.; Shaver, B. Semiconductor neutron detectors using depleted uranium oxide. In Proceedings of the Hard X-ray, gamma-ray, and neutron detector physics XVI, San Diego, USA, 2014.

10. Schoenes, J. J. Optical properties and electronic structure of UO2. Appl. Phys. 1978, 49, 1463.

11. Siekhaus W.; Crowhurst, J. Optical properties of a mechanically polished and air-equilibrated [111] UO2 surface by Raman and ellipsometric spectroscopy. In Proceedings of IOP Conference Series: Materials Science and Engineering, San Francisco, USA (12 - 17 July 2009).

12. Dugan, C.L.; Peterson, G.G.; Mock, A.; Young, C.; Mann, J.M.; Nastasi, M.; Schubert, M.; Wang, L.; Mei, W.-N.; Tanabe, I.; Dowben, P.A.; Petrosky, J.C. Electrical and material properties of hydrothermally grown single crystal (111) UO2. Eur. Phys. J. B. 2018, 91, 67.

13. Mock, A.; Dugan, C.L.; Knight, S.; Korlacki, R.; Mann, J.M.; Kimani, M.M.; Petrosky, J.C.; Dowben, P. A.; Schubert, M. Bandto-band transitions and critical points in the near-infrared to vacuum ultraviolet dielectric functions of single crystal urania and thoria. Appl. Phys. Lett. 2019, 21, 211901.

14. Meek, T.T.; Roedern, B. von; Clem, P.G.; Hanrahan Jr., R.J. Some optical properties of intrinsic and doped UO 2 thin films. Matl. Lett. 2005, 59, 1085- 1088.

15. Mo, C.; Yang, Y.; Kang, W.; Zhang, P. Electronic and optical properties of (U, Th) $\mathrm{O}_{2}$ compound from screened hybrid density functional studies. Phys. Lett. A. 2016, 380, 1481-1486.

16. Mann, M.; Thompson, D.; Serivalsatit, K.; Tritt, T. M.; Ballato, J.; Kolis, J. Hydrothermal Growth and Thermal Property Characterization of $\mathrm{ThO}_{2}$ Single Crystals. Cryst. Growth. Des. 2010, 2146-2151.

17. Young, C.; Petrosky, J.C.; Mann, J.M.; Hunt, E. M.; Turner, D.; Dowben, P.A. The lattice stiffening transition in UO $\mathrm{U}_{2}$ single crystals. J. Phys-Cond. Mat. 2017, 29, 035005.

18. Kelly, T.D.; Petrosky, J.C.; McClory, J.; Zens, T.; Turner, D.; Mann, J.M.; Kolis, J.; Colón Santana, J. A.; Dowben, P.A. The Debye Temperature for Hydrothermally Grown ThO ${ }_{2}$ Single Crystals. In Proceedings of MRS Symposium, 2013.

19. Kelly, T.; Petrosky, J.; Turner, D.; McClory, J.; Mann, J.; Kolis, J.; Zhang, X.; Dowben, P. The unoccupied electronic structure characterization of hydrothermally grown $\mathrm{ThO}_{2}$ single crystals. Phys. Status. Solidi. RRL. 2014, 283-286.

20. Turner, D. B.; Kelly, T. D.; Peterson, G. R.; Reding, J. D.; Hengehold, R. L.; Mann, J. M.; Kolis, J. W.; Zhang, X.; Dowben, P.A.; Petrosky, J. C. Electronic structure of hydrothermally synthesized single crystal $\mathrm{U}_{0.22} \mathrm{Th}_{0.78 \mathrm{O}}$. Phys. Status. Solidi. B 2016, 253, 1970-1976; doi: 10.1002/pssb.201600277

21. Young, C.; Petrosky, J.; Mann, J.M.; Hunt, E. M.; Turner, D.; Kelly, T. The work function of hydrothermally synthesized $\mathrm{UO}_{2}$ and the implications for semiconductor device fabrication. Phys. Status Solidi RRL 10 2016, 687-690.

22. Knight, S.; Korlacki, R.; Dugan, C.; Petrosky, J. C.; Mock, A.; Dowben, P.A.; Mann, J. M.; Kimani, M. M.; Schubert, M. Infrared-active phonon modes in single-crystal thorium dioxide and uranium dioxide. J. App. Phys. 2020, 127 - 125103; doi: $10.1063 / 1.5143724$. 
23. Lynds, L.; Young, W.; Mohl, J.; Libowitz, G. X-ray and Density Study of Nonstoichiometry in Uranium Oxides. Adv. Chem. Ser. 1963, 39, 58-65.

24. Leinders, G.; Cardinels, T.; Binnemans, K.; Verwerft, M. Accurate lattice parameter measurements of stoichiometric uranium dioxide. J. Nucl. Mater. 2015, 135-142.

25. Kresse, G.; Furthmüller. Efficient iterative schemes for ab initio total-energy calculations using a plane-wave basis set. J. Phys. Rev. B. 1996, 54, 11169.

26. Kresse, G.; Furthmüller. Efficiency of ab-initio total energy calculations for metals and semiconductors using a plane-wave basis set. J. Comput. Mater. Sci. 1996, 15-50.

27. Kresse, G.; Hafner, J. Theory of the crystal structures of selenium and tellurium: The effect of generalized-gradient corrections to the local-density approximation. Phys. Rev. B. 1993, 47-558.

28. Kresse, G.; Joubert, D. From ultrasoft pseudopotentials to the projector augmented-wave method. Phys. Rev. B 1999, 591758.

29. Perdew, J.P.; Burke, K.; Ernzerhof, M. Generalized Gradient Approximation Made Simple. Phys. Rev. Lett. 1996, 77-3865.

30. Heyd, J.; Scuseria, G. E.; Ernzerhof, M. Influence of the exchange screening parameter on the performance of screened hybrid functionals. J. Chem. Phys. 2003, 118-8207; Erratum: J. Chem. Phys. 124 (2006) 219906.

31. Ehrenreich, H.; Cohen, M. H. Self-Consistent Field Approach to the Many-Electron Problem. Phys. Rev. 1959, 115, 786-790.

32. Gajdos , M.; Hummer, K.; Kresse, G.; Furthmuller, J.; Bechstedt F. Linear optical properties in the projector-augmented wave methodology. Phys. Rev. B. 2006, 73, 045112.

33. Jayakumara, O.D.; Gopalakrishnana, I.K.; Vinub, A.; Asthanac, A.; Tyagia, A.K. Room temperature ferromagnetism in

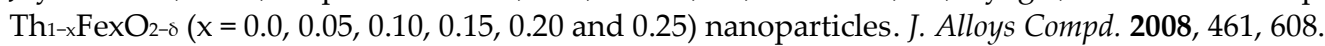

34. Szpunar, B.; Szpunar, J.A. Application of density functional theory in assessing properties of thoria and recycled fuels. J. Nuc. Mater. 2013, 439, 243-250.

35. Maehira, T.; Hotta, T. Relativistic band-structure calculations for electronic properties of actinide dioxides. J. Magn. Magn. Mater. 2007, 310, 754-756.

36. Kelly, P.J.; Brooks, M.S.S. Electronic structure and ground-state properties of the actinide dioxides. J. Chem. Soc. Faraday. T. 2. 1987, 83, 1189

37. Wen, X.-D.; Martin, R. L.; Roy, L. E.; Scuseria, G. E.; Rudin, S. P.; Batista, E. R.; McCleskey, T. M.; Scott, B. L.; Bauer, E.; Joyce, J.J.; Durakiewicz. Effect of spin-orbit coupling on the actinide dioxides $\mathrm{AnO}_{2}(\mathrm{An}=\mathrm{Th}, \mathrm{Pa}, \mathrm{U}, \mathrm{Np}, \mathrm{Pu}, \mathrm{and} \mathrm{Am}$ ): $\mathrm{A}$ screened hybrid density functional study. J. Chem. Phys. 2012, 137, 154707.

38. Baer, Y.; Schoenes J. Electronic structure and Coulomb correlation energy in $\mathrm{UO}_{2}$ single crystal. Solid State Commun. 1980, 33, 885.

39. Sui, P.F.; Dai, Z.H.; Zhang, X.L.; Zhao, Y.C. Electronic Structure and Optical Properties in Uranium Dioxide: the First Principle Calculations. Chin. Phys. Lett. 2015, 32, 077101.

40. Lasowski, R.; Madsen, G.K.H.; Blaha, P.; Schwarz, K. Magnetic structure and electric-field gradients of uranium dioxide: An ab initio study. Phys. Rev. B. 2004, 69, 140408R.

41. Joaquin, S. Relation between reference levels, work functions and contact potential differences in photoelectron spectroscopy. Chem. Phys. Lett. 1976, 42, 131-132. 